loss of function if it is compounded by recessive mutation or monoallelic exclusion of the remaining allele. A genomic rearrangement may also disrupt regulatory elements that influence the expression of neighboring genes; thus, in some cases, a gene related to autism may lie adjacent to the deletion or duplication.

Our findings have implications for an understanding of the genetic basis for ASDs. An important feature of the de novo CNVs we report is that each is individually rare in the population of patients. None of the genomic variants we detected were observed more than twice in our sample, and most were seen but once. Although our sample size is small, these results suggest that lesions at many different loci can contribute to autism, a result consistent with the findings from cytogenetics, as well as consistent with the failure to find common heritable variants with a major effect on disease risk. Lack of recurrence may in fact reflect an underlying reality that autistic behavior can result from many different genetic defects. This would be consistent with the hypothesis that the common features of autism such as failure to develop social skills and repetitive and obsessive behavior may in fact be the consequence of a reaction to many different cognitive impairments, drawing their "commonality" from a normal but maladaptive programmed response of humans early in development to those diverse impairments.

We do not know the full contribution of spontaneous mutation to autism. Population studies divide autism into sporadic and familial or "multiplex." Our work provides clear evidence that these two classes are indeed genetically distinct. The rate of de novo mutation in multiplex families was significantly lower than for sporadic cases (Table 2, $P=0.04$ ), as would be expected if there were two different genetic mechanisms contributing to risk: spontaneous mutation and inheritance, with the latter being more frequent in families that have multiple affected children.

The rate of spontaneous mutation that we detect in autism is an underestimate. Adding the known rate of cytogenetically visible abnormalities, the total frequency of de novo variation detectable in sporadic cases is $\sim 15 \%$ at our current resolution. Because of the limited resolution of genome microarray scans, we expect that we fail to detect the vast majority of CNVs. Much smaller deletions or even point mutations can produce the same consequences as the larger, more easily detectable events. As technology for discovering spontaneous germline mutation in children improves, the proportion of autism cases with detectable events is bound to rise.

We can incorporate a high rate of spontaneous mutation in a genetic model that accounts for both sporadic and familial forms of the disease, based on new mutations that cause autism by haploinsufficiency but have incomplete penetrance, especially in females. Such individuals who escape the phenotypic consequences can then pass on the mutation in an apparently dominant fashion to their children. This model makes very clear predictions that can be tested in the short term.

Our findings highlight how methods for directly detecting CNVs genomewide provide a powerful alternative to traditional gene-mapping approaches for discovering genetic risk factors in autism and in other disorders of complex etiology. Improved technologies for mutation detection, such as high-throughput DNA sequencing and tiling-resolution oligonucleotide arrays, promise to improve our power to identify new mutations associated with disease.

\section{References and Notes}

1. E. Fombonne, J. Clin. Psychiatry 66 (suppl. 10), 3 (2005).

2. S. M. Klauck, Eur. J. Hum. Genet. 14, 714 (2006).

3. J. A. Vorstman et al., Mol. Psychiatry 11, 1 (2006).

4. R. E. Amir et al., Nat. Genet. 23, 185 (1999).

5. European Chromosome 16 Tuberous Sclerosis Consortium, Cell 75, 1305 (1993).

6. J. K. Pritchard, Am. J. Hum. Genet. 69, 124 (2001).

7. L. Feuk, A. R. Carson, S. W. Scherer, Nat. Rev. Genet. 7, 85 (2006).

8. J. Sebat et al., Science 305, 525 (2004).

9. A. J. lafrate et al., Nat. Genet. 36, 949 (2004).

10. D. A. Koolen et al., Nat. Genet. 38, 999 (2006)

11. A. J. Sharp et al., Nat. Genet. 38, 1038 (2006).

12. C. Shaw-Smith et al., Nat. Genet. 38, 1032 (2006).

13. C. Shaw-Smith et al., J. Med. Genet. 41, 241 (2004).

14. L. E. Vissers et al., Am. J. Hum. Genet. 73, 1261 (2003).

15. M. L. Jacquemont et al., J. Med. Genet. 43, 843 (2006).

16. R. Lucito et al., Genome Res. 13, 2291 (2003).

17. J. N. Ferguson et al., Nat. Genet. 25, 284 (2000).

18. M. Kosfeld, M. Heinrichs, P. J. Zak, U. Fischbacher, E. Fehr, Nature 435, 673 (2005).

19. M. Ghaziuddin, M. Burmeister, J. Autism Dev. Disord. 29, 259 (1999).

20. M. A. Manning et al., Pediatrics 114, 451 (2004).

21. J. Veenstra-VanderWeele, E. H. Cook Jr., Mol. Psychiatry 9, 819 (2004)
22. C. M. Durand et al., Nat. Genet. 39, 25 (2007).

23. T. Igarashi et al., J. Am. Soc. Nephrol. 12, 713 (2001).

24. K. Bhalla et al., J. Hum. Genet. 49, 308 (2004).

25. K. Huebner, C. M. Croce, Nat. Rev. Cancer 1, 214 (2001).

26. D. I. Smith, Y. Zhu, S. McAvoy, R. Kuhn, Cancer Lett. 232 48 (2006).

27. S. M. Gospe Jr. et al., Neurology 39, 1277 (1989).

28. E. Orye, M. J. Delbeke, B. Vandenabeele, Lancet 2, 1376 (1971).

29. D. Viskochil et al., Cell 62, 187 (1990)

30. W. J. Kent et al., Genome Res. 12, 996 (2002).

31. We wish to thank patients and families for their valuable contributions and the NIMH Center for Collaborative Genetic Studies of Mental Disorders, the Autism Genetic Resource Exchange (AGRE), the Centre d'Etude du Polymorphisme Humain (CEPH), and D. Levy for providing materials or data for the study. We are grateful to G. Fischbach, C. Lord, N. Heinz, E. Cook, L. Brzustowicz, J. and M. Simons, and L. lakoucheva for helpful discussions throughout the study, and we thank C. Reed, P. Roccanova, J. Lloyd, V. Grubor, L. Rodgers, D. Esposito, L. Hufnagel, X. Zhao, E. Thorolfsdottir, K. A. Olafsdottir, and the staff of Lingen EHF for technical assistance. This work was supported by a grant from the Simons Foundation and by NIMH grant MH076431 to ].S., which reflects cofunding from Autism Speaks, Cure Autism Now, and the Southwestern Autism Research and Resource Center. Additional support was provided by NIMH grant MH61009 to J.S.S., grants to D.S. from Nancy Lurie Marks Family Foundation and the National Alliance for Autism Research (NAAR), and a grant to K.P. and T.L. from the University of Tampere Hospital Medical Fund. AGRE is a program of Cure Autism Now and is supported in part by NIMH grant MH64547 to D.H.G.

\section{Supporting Online Material}

www.sciencemag.org/cgi/content/full/1138659/DC1

Materials and Methods

Tables S1 to S3

References

11 December 2006; accepted 6 March 2007

Published online 15 March 2007;

10.1126/science. 1138659

Include this information when citing this paper.

\title{
Raman-Assisted Crystallography Reveals End-0n Peroxide Intermediates in a Nonheme Iron Enzyme
}

\author{
Gergely Katona, ${ }^{1}$ Philippe Carpentier, ${ }^{1}$ Vincent Nivière, ${ }^{2}$ Patricia Amara, ${ }^{1}$ Virgile Adam, ${ }^{3}$ \\ Jérémy Ohana, ${ }^{1}$ Nikolay Tsanov, ${ }^{1}$ Dominique Bourgeois ${ }^{1,3 *}$
}

Iron-peroxide intermediates are central in the reaction cycle of many iron-containing biomolecules. We trapped iron(III)-(hydro)peroxo species in crystals of superoxide reductase (SOR), a nonheme mononuclear iron enzyme that scavenges superoxide radicals. X-ray diffraction data at 1.95 angstrom resolution and Raman spectra recorded in crystallo revealed iron-(hydro)peroxo intermediates with the (hydro)peroxo group bound end-on. The dynamic SOR active site promotes the formation of transient hydrogen bond networks, which presumably assist the cleavage of the iron-oxygen bond in order to release the reaction product, hydrogen peroxide.

$\mathrm{T}$ The interaction of dioxygen with ironcontaining proteins is important in many biological processes, including transport, metabolism, respiration, and cell protection. The reaction of oxygen or its reduced derivatives, superoxide and hydrogen peroxide, with iron enzymes often involves short-lived iron-peroxide intermediates along the reaction cycle $(1,2)$.
Heme-based peroxidases, catalases, and many oxygenases promote heterolytic cleavage of the peroxide oxygen-oxygen bond to form highvalence reactive iron-oxo species. In contrast, other iron enzymes, such as superoxide reductase (SOR) $(3,4)$, are fine-tuned to cleave the ironoxygen bond and avoid the formation of potentially harmful iron-oxo species. Although the 
protein, the metal configuration, and the solvent environment have been shown to play a role, the mechanisms by which iron-peroxide intermediates are processed are not fully understood $(1,2)$. Despite pioneering studies on heme proteins (5-7), structural data revealing peroxide species in nonheme mononuclear iron enzymes have remained scarce $(8)$. We have developed an approach in which kinetic crystallography (9) was assisted by "in crystallo" Raman spectroscopy (10) to characterize (hydro)peroxo species in SOR. SOR is found in some air-sensitive bacteria and archaea and converts the toxic superoxide anion radical $\left(\mathrm{O}_{2}{ }^{-}\right)$into hydrogen peroxide $\left(\mathrm{H}_{2} \mathrm{O}_{2}\right)$ via a one-electron reduction pathway: $\mathrm{O}_{2}{ }^{\cdot-}+2 \mathrm{H}^{+}+\mathrm{SOR}\left(\mathrm{Fe}^{2+}\right) \rightarrow \mathrm{H}_{2} \mathrm{O}_{2}+\mathrm{SOR}\left(\mathrm{Fe}^{3+}\right)$ $(3,4,11)$. The SOR catalytic domain displays an immunoglobulin-like fold $(12,13)$ encompassing an iron atom coordinated to four equatorial histidines and one axial cysteine, thus bearing structural resemblance to the ubiquitous cytochromes P450s. However, contrary to P450s, the ferrous enzyme is stable under atmospheric conditions, with a vacant, solvent-exposed, sixth coordination site where $\mathrm{O}_{2}{ }^{--}$is thought to bind (14). Investigations of various SOR adducts $(12,14,15)$, pulse-radiolysis studies (16-18), and resonance Raman spectroscopy experiments $(19,20)$ have suggested an inner-sphere catalytic mechanism involving the formation of transient iron(III)-

${ }^{1}$ Institut de Biologie Structurale (IBS) Jean-Pierre Ebel, Commissariat à l'Energie Atomique (CEA), Centre National de la Recherche Scientifique (CNRS), Université Joseph Fourier, 41 rue Jules Horowitz, F-38027 Grenoble, France. ${ }^{2}$ Laboratoire de Chimie et Biologie des Métaux, Institut de Recherches en Technologies et Sciences pour le Vivant, CEA, CNRS, Université ]. Fourier, UMR 5249, 17 rue des Martyrs, 38054 Grenoble Cedex 9, France. ${ }^{3}$ European Synchrotron Radiation Facility (ESRF), 6 rue Jules Horowitz, BP 220, 38043 Grenoble Cedex, France.

*To whom correspondence should be addressed. E-mail: dominique.bourgeois@ibs.fr (hydro)peroxo species. As described for similar enzymes $(2,21)$, protonation steps play a crucial role in governing the chemistry that occurs at the SOR active site $(11,16,17)$. In SOR from the sulfate-reducing bacterium Desulfoarculus baarsii $(4,12)$, a first iron(III)-peroxo intermediate has been proposed to be rapidly protonated (in $\sim 100$ $\mu \mathrm{s})$, forming a more stable iron(III)-hydroperoxo species (17). A second protonation then occurs, possibly promoted by a water molecule (22), and yields the $\mathrm{H}_{2} \mathrm{O}_{2}$ product through a dissociative mechanism in which $\mathrm{Glu}^{47}$ ultimately binds to the oxidized enzyme $(13,17)$. Thus, SOR avoids heterolytic cleavage of the $\mathrm{O}-\mathrm{O}$ bond, preventing the formation of oxo-ferryl compounds. To date, the structure of the iron-peroxide species that can be accommodated within the SOR active site and the mechanism governing the decisive second protonation step have remained elusive (11). The structural data described below reveal a series of end-on iron(III)-(hydro)peroxo species involved in tight hydrogen bond networks (Fig. 1) and allow us to propose a mechanism for protonassisted release of $\mathrm{H}_{2} \mathrm{O}_{2}$ in SOR.

Table 1. Geometry of the active site. Fe distance from the His plane was defined by the coordinating $\mathrm{N}$ atoms of the equatorial histidines in $\AA$. Increasing value indicates an iron position closer to Cys ${ }^{116}$.

\begin{tabular}{lccc} 
WT-SOR & $\begin{array}{c}\text { E114A-SOR } \\
\text { reduced }\end{array}$ & $\begin{array}{c}\text { E114A-SOR } \\
\text { peroxide } \\
\text { intermediates }\end{array}$ & $\begin{array}{c}\text { DFT } \\
\text { calculation }\end{array}$ \\
\hline
\end{tabular}

\begin{tabular}{|c|c|c|c|c|}
\hline \multicolumn{5}{|c|}{ Monomer A } \\
\hline Fe-S $(\AA)$ & 2.4 & 2.4 & 2.5 & \\
\hline Fe from His plane $(\AA)$ & 0.4 & 0.4 & 0.3 & \\
\hline \multicolumn{5}{|c|}{ Monomer $B$} \\
\hline $\mathrm{Fe}-\mathrm{S}(\AA)$ & 2.4 & 2.4 & 2.5 & 2.48 \\
\hline $\mathrm{Fe}-01(\AA)$ & & & 2.0 & 2.19 \\
\hline Fe-01-02 $\left(^{\circ}\right)$ & & & 126 & 125 \\
\hline $\mathrm{C}_{\beta}-\mathrm{S}-01-02\left(^{\circ}\right)$ & & & 140 & 168 \\
\hline Fe from His plane $(\AA)$ & 0.4 & 0.4 & 0.3 & 0.10 \\
\hline \multicolumn{5}{|c|}{ Monomer C } \\
\hline $\mathrm{Fe}-\mathrm{S}(\AA)$ & 2.4 & 2.5 & 2.5 & 2.44 \\
\hline $\mathrm{Fe}-01(\AA)$ & & & 2.0 & 1.94 \\
\hline Fe-01-02 $\left(^{\circ}\right)$ & & & 126 & 123 \\
\hline $\mathrm{C}_{\beta}-\mathrm{S}-01-02\left(^{\circ}\right)$ & & & 132 & 114 \\
\hline Fe from His plane $(\AA)$ & 0.5 & 0.4 & 0.3 & 0.16 \\
\hline \multicolumn{5}{|c|}{ Monomer D } \\
\hline Fe-S (Å) & 2.4 & 2.5 & 2.6 & 2.49 \\
\hline Fe-01 (̊) & & & 2.0 & 2.22 \\
\hline Fe-01-02 $\left(^{\circ}\right)$ & & & 122 & 123 \\
\hline $\mathrm{C}_{\beta}-\mathrm{S}-01-02\left(^{\circ}\right)$ & & & 112 & 99 \\
\hline Fe from His plane $(\AA)$ & 0.4 & 0.3 & 0.0 & 0.11 \\
\hline
\end{tabular}

Fig. 1. Structural overview of SOR. The $x$-ray structure of the SOR-E114A homodimer in the native reduced state is shown as magenta (monomer A) and cyan (monomer B) ribbons with the exception of the LID loop (residues 45 to 49 ), which is colored in dark green and orange for monomers $A$ and $B$, respectively. Reduced and oxidized iron atoms are shown as green and orange balls, respectively. (Inset) The active site of monomer $B$ upon addition of $\mathrm{H}_{2} \mathrm{O}_{2}$. The residues coordinating the active iron $\left(\mathrm{His}^{49}, \mathrm{His}^{69}\right.$, $\mathrm{His}^{75}, \mathrm{His}^{119}$, and $\mathrm{Cys}^{116}$ ) as well as Lys $^{48}$ are represented as sticks. The bound peroxide ligand is shown as a red stick. Water molecules are shown as red balls. In order to support the diatomic nature of the peroxide intermediate, simulated annealed $F_{\text {obs }}$ $F_{\text {calc }}$ maps omitting the distal or proximal oxygens of the 0-0 moiety, respectively, were calculated. The two maps are displayed in green (distal) and orange (proximal) at a contour level of 3.0 $\sigma$.

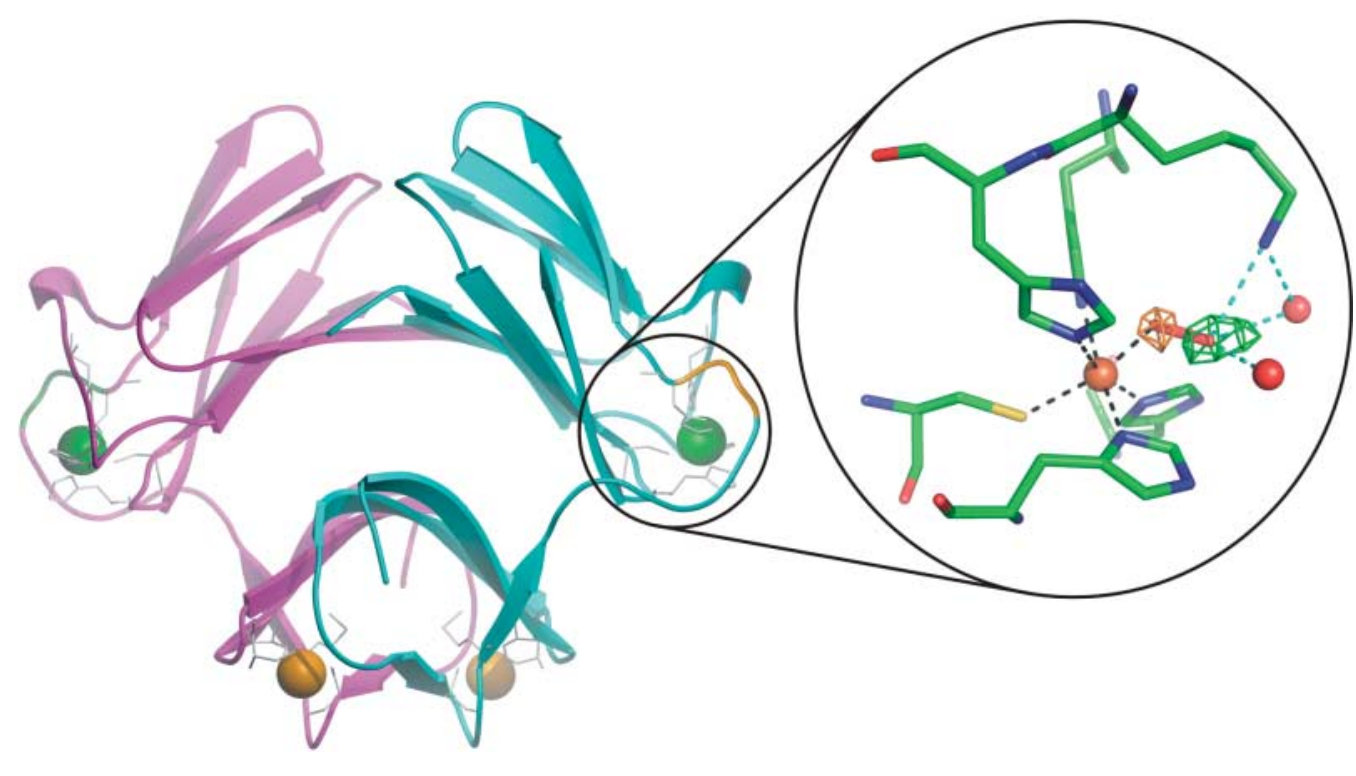


Mononuclear iron-peroxide complexes are generally obtained by reacting iron(II) with excess $\mathrm{H}_{2} \mathrm{O}_{2}$ (23). However, to minimize Fenton-driven generation of toxic hydroxyl radicals, we first oxidized crystalline SOR with hexachloroiridate (IV) and then exposed it to $\mathrm{H}_{2} \mathrm{O}_{2}$ for 3 min before freezing (24). Because the isolation of iron(III)- peroxide complexes is hampered by their high reactivity, crystallographic data were collected with the mutant enzyme E114A $\left(\mathrm{Glu}^{114} \rightarrow \mathrm{Ala}^{114}\right)$, in which, as described for the E47A $\left(\mathrm{Glu}^{47} \rightarrow \mathrm{Ala}^{47}\right)$ variant $(19,20)$, these intermediates are stabilized [Supporting Online Material (SOM) text]. Comparison of the native crystal structures of the wild-
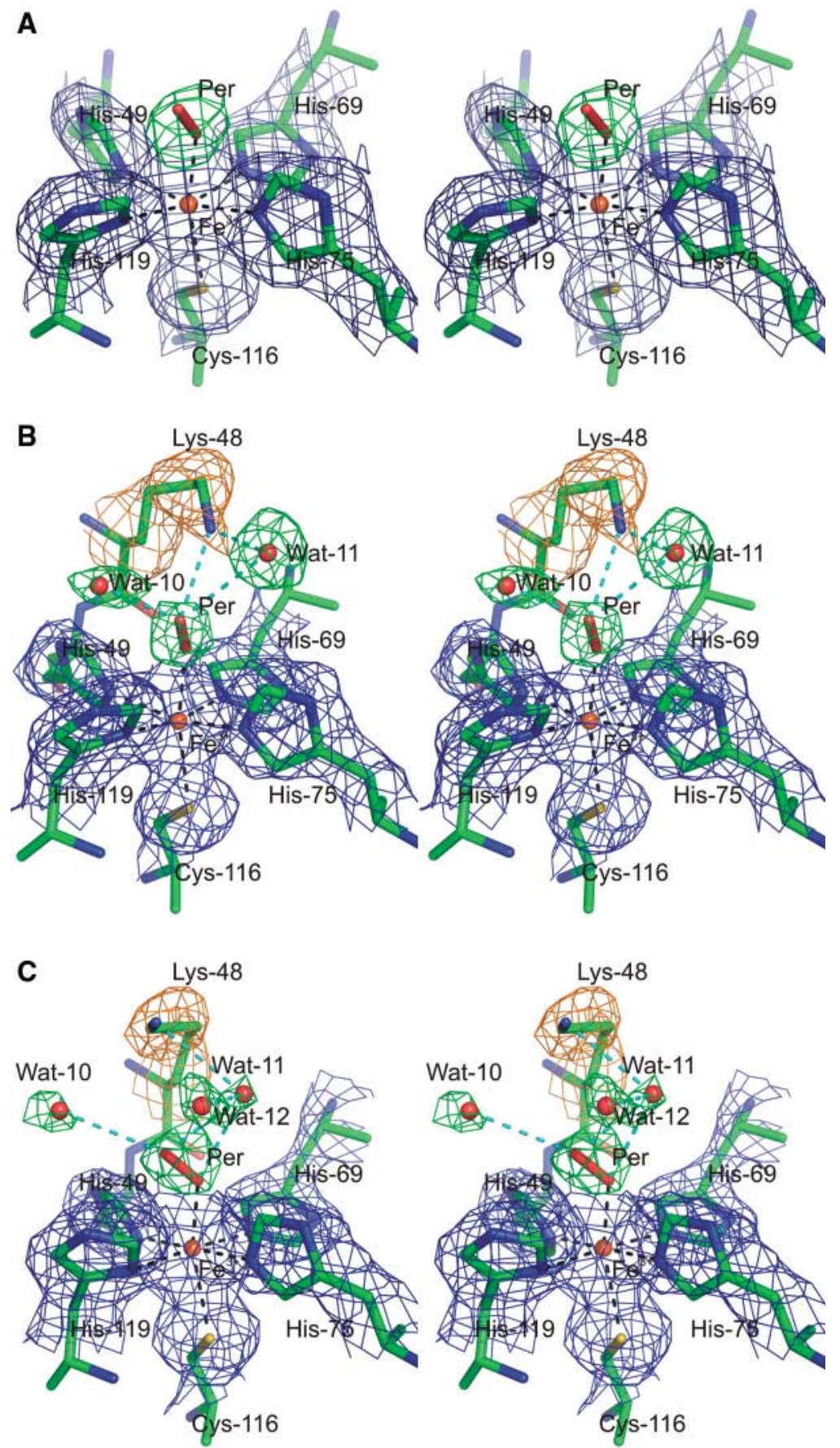

Fig. 2. Structure of the SOR-peroxide intermediates. Stereo views of the peroxide-bound SOR active sites in monomers $C, B$, and $D$ are shown in $(\mathbf{A}),(\mathbf{B})$, and $(\mathbf{C})$, respectively. Final $2 F_{\text {obs }}-F_{\text {calc }}$ maps (blue, contoured at $1.0 \sigma$ ), simulated annealed $F_{\text {obs }}-F_{\text {calc }}$ maps omitting the peroxo moiety and associated water molecules (green, contoured at $4.5 \sigma$ ), and simulated annealed $F_{\text {obs }}-F_{\text {calc }}$ maps omitting only Lys ${ }^{48}$ (orange, contoured at $3.5 \sigma$ ) are shown, overlaid on the refined models of the SOR-peroxide intermediates. Hydrogen bonds and iron coordination are shown as blue and black dashed lines, respectively.

type and mutant enzymes revealed that the loss of the E114 side chain does not alter the overall enzyme structure (Table 1).

The asymmetric unit in SOR-E114A crystals contains four monomers, denoted A to D. Upon soaking with $\mathrm{H}_{2} \mathrm{O}_{2}$, diffraction data to $1.95 \AA$ resolution (24) (table S1) revealed elongated features in the electron density maps that are consistent with the formation of end-on $\left(\eta^{1}\right)$ peroxide species in monomers B, C, and D (Figs. 1 and 2), whereas monomer A did not react. To verify the chemical nature of the observed species, we developed a Raman spectrometer to analyze cryocooled crystals under conditions identical to those used for x-ray data collection $(24,25)$ (fig. $\mathrm{S} 1)$. Upon $\mathrm{H}_{2} \mathrm{O}_{2}$ treatment, two ${ }^{18} \mathrm{O}$ isotopesensitive main bands at $\sim 567 \mathrm{~cm}^{-1}$ and $\sim 838 \mathrm{~cm}^{-1}$ appeared in the Raman spectra of SOR crystals (Fig. 3). Although these bands probably involve the coupling of a number of vibrational modes, they fall within the expected range for $v(\mathrm{Fe}-\mathrm{OO}(\mathrm{H}))$ and $v(\mathrm{O}-\mathrm{O})$ frequencies of iron-peroxide species, respectively (26) (SOM text). Importantly, they are not specific to the crystalline phase, because they also appeared with solution samples similarly treated with hydrogen peroxide (SOM text). In addition, Raman spectra from crystals exposed to X-rays (27) showed the same signature as unexposed crystals, ruling out the possibility of substantial photo-alteration during data collection [it is known that the solvent-exposed SOR active site is sensitive to reduction by x-rayinduced photo-electrons (12)]. Overall, in crystallo Raman spectra strongly suggested the buildup of iron-peroxide species in the crystal. To assess the protonation state of these species, we performed density functional theory (DFT) calculations (SOM text) on model SOR active sites based on the x-ray structures determined in this work. In monomers $\mathrm{B}$ and $\mathrm{D}$, these calculations favor highspin $\eta^{1}$ hydroperoxo species that are protonated at the distal oxygen, consistent with pulse-radiolysis studies that suggested rapid protonation of the $\mathrm{SOR}$ iron-peroxo species even at the basic $\mathrm{pH}$ $(\mathrm{pH}=9)$ used in our work (17). In monomer C, an $\eta^{1}$ species is also favored, but its protonation state cannot be firmly established.

Final x-ray models of SOR monomers B, C, and D show end-on iron(III)-peroxide species in three different configurations that all display the distal oxygen pointing toward His ${ }^{119}$ to accommodate steric constraints imposed by the protein matrix. Thus, the atoms $\mathrm{C}_{\beta, \text { Cys116 }}-\mathrm{S}_{\gamma, \text { Cys116 }}-\mathrm{Fe}-\mathrm{O}-\mathrm{O}$ are non-coplanar, resulting in nonoptimal $\pi$ orbital overlap and contributing to weaken the iron-oxygen bond, as also suggested by the long Fe-O distances found in our DFT calculations (Table 1).

In SOR, a solvent-exposed flexible loop (residues 45 to 49 , called "LID" thereafter) is located near the active site and includes $\mathrm{Lys}^{48}$, an evolutionary conserved residue critical for efficient catalysis $(16,17)$. The formation of ironperoxide species modified the conformation of Lys $^{48}$ relative to that in the native reduced state (SOM text). In monomer $\mathrm{C}$, the hydroperoxo 
moiety only interacts with the active iron, and the LID loop displays a "locked-open" conformation, possibly because of weak crystal lattice contacts (SOM text). This LID conformation prevents $\mathrm{Lys}^{48}$ from interacting with the hydroperoxo moiety, leaving the side chain of this residue in a disordered state. In contrast, in monomer B, the LID loop is found "locked closed," and Lys ${ }^{48}$ facilitates a tight hydrogen bond network around the distal oxygen of the peroxide moiety that also includes two water molecules (Wat ${ }^{10}$ and $\mathrm{Wat}^{11}$ ) (Fig. 2). The positively charged amino group of Lys ${ }^{48}$ (SOM text) attracts the peroxide ligand, presumably inducing a stretch of the $\mathrm{S}_{\gamma, \text { Cys116 }}$-Fe-O-O moiety that may further weaken the $\mathrm{Fe}-\mathrm{O}$ bond. In monomer $\mathrm{D}$, the side chain of Lys ${ }^{48}$ slightly rotates away from the hydroperoxo moiety, and the
Fig. 3. Raman spectra of SOR crystals. After reaction with $\mathrm{H}_{2} \mathrm{O}_{2}$, the E114A SOR mutant reveals bands at $\sim 567 \mathrm{~cm}^{-1}$ and $\sim 838 \mathrm{~cm}^{-1}$, which are isotopically shifted to $\sim 563 \mathrm{~cm}^{-1}$ and $\sim 802 \mathrm{~cm}^{-1}$ in the presence of $\mathrm{H}_{2}{ }^{18} \mathrm{O}_{2}$ (vertical gray lines). Similar Raman bands and ${ }^{18} \mathrm{O}$ isotopic shifts are observed in solution experiments (fig. S2). E114A-SOR in the native reduced form does not exhibit these bands; neither

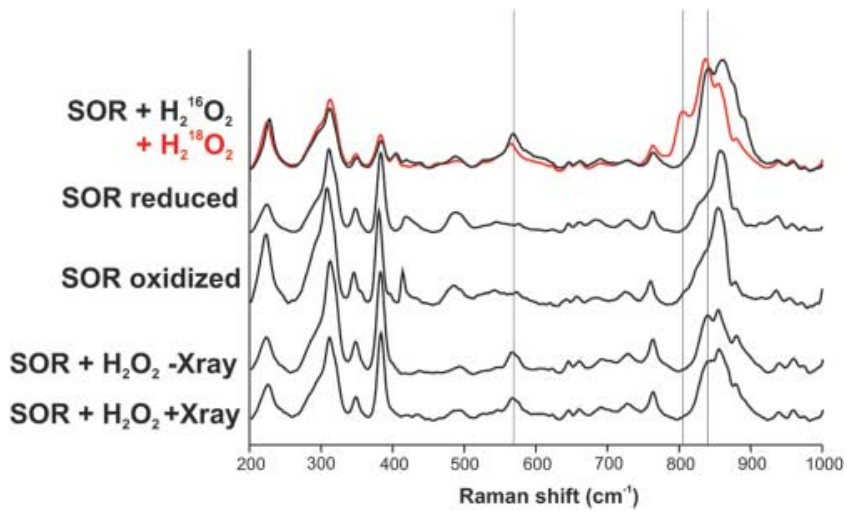
do crystals oxidized by hexachloroiridate(IV). The peaks at $\sim 567 \mathrm{~cm}^{-1}$ and $\sim 838 \mathrm{~cm}^{-1}$ are not substantially affected by exposure to an $\mathrm{x}$-ray dose of $3 \times 10^{5} \mathrm{~Gy}$, which is about the same dose as used for data collection. two water molecules $\mathrm{Wat}^{10}$ and $\mathrm{Wat}^{11}$ are still observed, together with a third molecule $\left(\mathrm{Wat}^{12}\right)$ that may play a stabilizing role. However, Wat ${ }^{10}$ now forms a hydrogen bond with the amino group of $\mathrm{Ala}^{45}$, whereas $\mathrm{Wat}^{11}$ moves slightly toward the iron so that it interacts with both proximal and distal oxygen atoms of the hydroperoxo moiety. Wat ${ }^{11}$ is therefore in a favorable position to donate a proton to the proximal oxygen atom, allowing the formation and release of hydrogen peroxide. This is a crucial step that differentiates SOR from heme enzymes where protonation occurs at the distal oxygen, liberating water and oxo-ferryl species (2). Simultaneously, a combination of subtle rearrangements of the iron-coordinating histidines shifts the iron into the plane defined by the four equatorial coordinating nitrogens (Table 1) (28). This conformation possibly facilitates access of $\mathrm{Wat}^{11}$ to the metal and the proximal oxygen.

Our data highlight the dynamic behavior of the SOR active site en route to product formation (Fig. 4 and movie S1). Monomer C may be viewed as an early state along the reaction coordinate that precedes the conformational rearrangements leading to the protonation of the $\mathrm{HOO}^{-}$adduct. We suggest that this state is sta-
Fig. 4. Proposed mechanism of superoxide reduction by SOR. The proposed catalytic cycle begins with the reduced pentacoordinated active site (1). Superoxide binds (2) and gets reduced (red arrow) to an unprotonated peroxo species (3). Previous data suggested that this intermediate could correspond to a high-spin side-on peroxo- $\mathrm{Fe}^{3+}$ species $(19,20)$ (SOM text). The first protonation step leads to the configuration observed in monomer C (4). Lys $^{48}$ and two water molecules are recruited (5), yielding the configuration observed in monomer B that subsequently rearranges to give the configuration of monomer D (6). At this point, protonation of the proximal oxygen atom (red arrow) is facilitated by the key water molecule Wat ${ }^{11}$. Hydrogen peroxide is formed and leaves the active site with the assistance of the hydroxide ion product (7). After a rearrangement of the LID loop, $\mathrm{Glu}^{47}$ replaces the hydroxide ion (8). Lastly, the active site is regenerated to its reduced state (1) by an unknown external factor. Along the catalytic cycle, blue color indicates the (hydro)peroxo species.
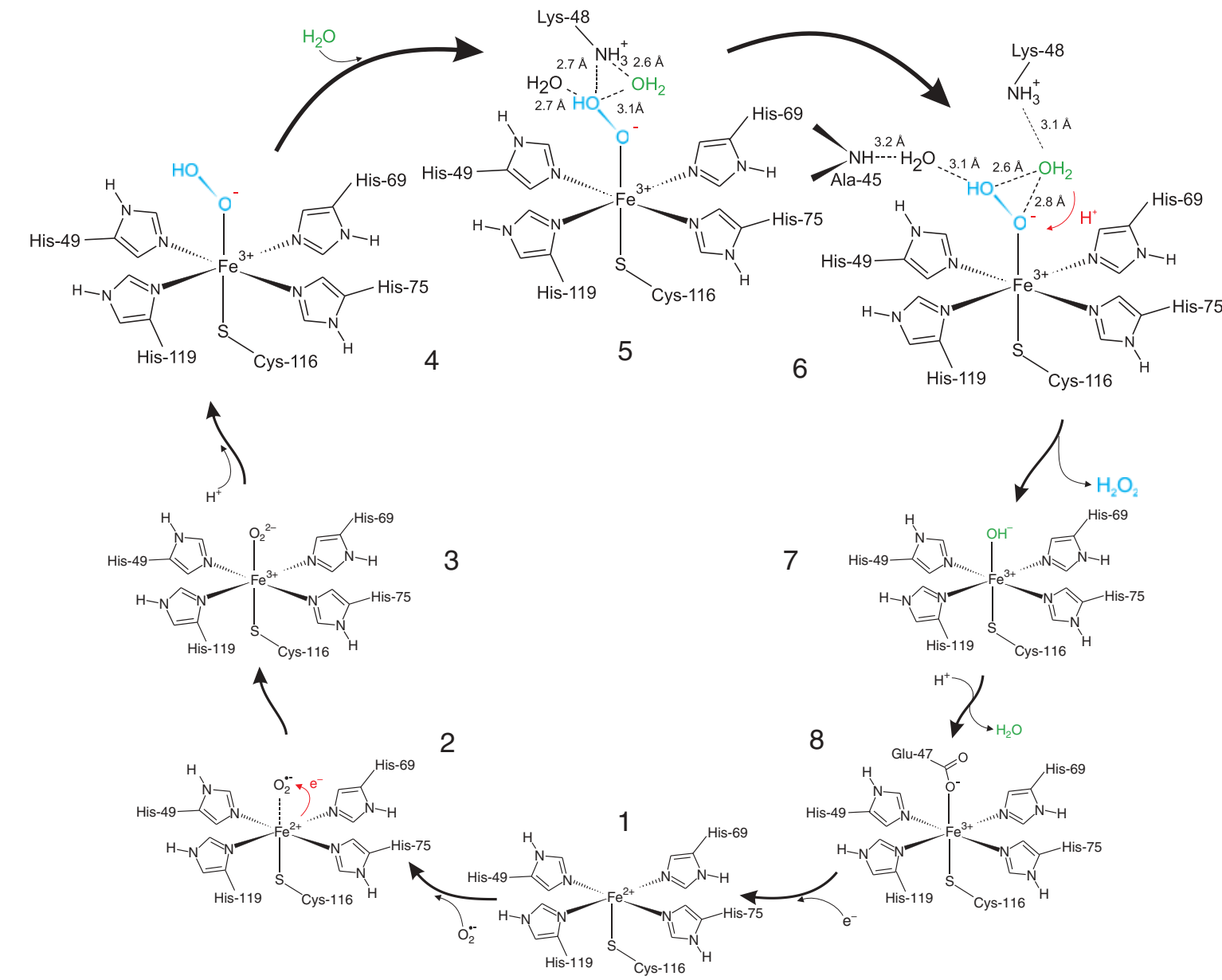
bilized in the crystal because of the locked-open configuration of the LID loop. In contrast, monomers $\mathrm{B}$ and $\mathrm{D}$ reveal subsequent activated configurations, emphasizing the catalytic role of $\mathrm{Lys}^{48}$. The observation of $\mathrm{Wat}^{11}$ in the immediate environment of the hydroperoxo species in these monomers strongly supports the hypothesis that this water molecule is the proton donor for product formation and release. We propose that Lys ${ }^{48}$ hydrogen bonds to Wat ${ }^{11}$ and imports it into the SOR active site in a motion promoted by electrostatic attraction of the positively charged amino group to the hydroperoxo ligand. Once anchored in the vicinity of the proximal oxygen, Wat ${ }^{11}$ becomes more acidic because of the interaction with the amino group of Lys ${ }^{48}$. Protonation of the proximal oxygen is probably simultaneous with the cleavage of the iron-oxygen bond, and Wat ${ }^{11}$ may immediately replace the hydrogen peroxide product in the form of a hydroxide ion until $\mathrm{Glu}^{47}$ binds to the iron, as already suggested $(18,22)$.

SOR illustrates the key role played by subtle protein motions in enzyme catalysis (29). In crystalline SOR, the flexible LID loop adopts various conformations, suggesting that there is little free energy difference between disordered (entropy-driven) states for this loop and ordered (enthalpy-driven) ones where Lys ${ }^{48}$ is stabilized by transient H-bonding networks. Our data are consistent with the idea of a breathing of the LID loop that serves to import catalytically competent water molecules into the SOR active site (29). In the crystal, local packing forces may slightly modify the thermodynamic energy balance, selecting different conformations in each monomer.

The structural observations described in this work are obviously not sufficient to entirely account for the specific reactivity of SOR toward breakage of the iron-oxygen bond. Finely tuned electron donation by the trans thiolate ligand $\left(\mathrm{Cys}^{116}\right)$ is expected to precisely adjust the strength of this bond (11). Furthermore, several lines of evidence indicate that the iron spin state greatly modulates the strength of the iron-oxygen and oxygen-oxygen bonds in iron(III)-peroxide complexes $(1,11,21,23)$. Whereas many heme catalysts that promote cleavage of the O-O bond involve low-spin states of the iron, SOR $(19,20,30)$ and the oxygen carrier di-iron hemerythrin (31) are unique in that they involve a high-spin $(S=5 / 2)$ iron state (30) (SOM text). Interestingly, SOR and hemerythrin share structural and spectroscopic properties: In oxy-hemerythrin, an end-on iron-peroxide species stabilized by a strong hydrogen bond is also observed (31). In addition, the Raman vibrations measured for SOR and oxy-hemerythrin are relatively similar and imply a weaker Fe-O bond and a stronger O-O bond when compared to low-spin iron-peroxide model compounds known to favor heterolytic cleavage of the O-O bond (26).

The data suggest a possible mechanism for hydrogen peroxide formation, highlighting the role of a key water molecule finely controlled by the enzyme dynamics. The revealed conforma- tional transitions provide a strong basis for further computational and structural investigations of the mechanism of superoxide scavenging by SOR and may facilitate the design of biomimetic catalysts.

\section{References and Notes}

1. M. Costas, M. P. Mehn, M. P. Jensen, L. Que, Chem. Rev. 104, 939 (2004).

2. I. G. Denisov, T. M. Makris, S. G. Sligar, I. Schlichting, Chem. Rev. 105, 2253 (2005).

3. F. E. Jenney Jr., M. F. J. M. Verhagen, X. Cui, M. W. W. Adams, Science 286, 306 (1999).

4. M. Lombard, D. Touati, M. Fontecave, V. Niviere, J. Biol. Chem. 275, 27021 (2000).

5. G. I. Berglund et al., Nature 417, 463 (2002).

6. I. Schlichting et al., Science 287, 1615 (2000).

7. K. Kuhnel, E. Derat, ]. Terner, S. Shaik, I. Schlichting, Proc. Natl. Acad. Sci. U.S.A. 104, 99 (2007).

8. A. Karlsson et al., Science 299, 1039 (2003).

9. D. Bourgeois, A. Royant, Curr. Opin. Struct. Biol. 15, 538 (2005).

10. P. R. Carey, J. Dong, Biochemistry 43, 8885 (2004).

11. L. M. Brines, ]. A. Kovacs, Eur. J. Inorg. Chem. 2007, 29 (2007).

12. V. Adam, A. Royant, V. Niviere, F. P. Molina-Heredia D. Bourgeois, Structure 12, 1729 (2004).

13. A. P. Yeh, Y. Hu, F. E. Jenney Jr., M. W. Adams, D. C. Rees, Biochemistry 39, 2499 (2000).

14. M. D. Clay et al., J. Am. Chem. Soc. 124, 788 (2002).

15. M. D. Clay et al., Biochemistry 45, 427 (2006).

16. J. P. Emerson, E. D. Coulter, D. E. Cabelli, R. S. Phillips, D. M. Kurtz Jr., Biochemistry 41, 4348 (2002).

17. V. Niviere et al., Biochemistry 43, 808 (2004).

18. J. V. Rodrigues, I. A. Abreu, D. Cabelli, M. Teixeira, Biochemistry 45, 9266 (2006).

19. C. Mathe et al., J. Am. Chem. Soc. 124, 4966 (2002).

20. C. Mathe, V. Niviere, C. Houee-Levin, T. A. Mattioli, Biophys. Chem. 119, 38 (2006).

21. G. H. Loew, D. L. Harris, Chem. Rev. 100, 407 (2000).

22. C. Mathe, V. Niviere, T. A. Mattioli, J. Am. Chem. Soc. 127, 16436 (2005).

23. G. Roelfes et al., Inorg. Chem. 42, 2639 (2003).

24. Materials and methods are available as supporting material on Science Online.

25. Raman experiments were performed under nonresonant conditions, taking advantage of the large protein concentration found in crystals to enhance the signal-to-noise ratio. In this way, the crystals were sampled homogeneously, and potential light-induced damage or photochemistry that could develop under resonant conditions was avoided.

26. J.-]. Girerd, F. Banse, A. ]. Simaan, in Metal-Oxo and Metal-Peroxo Species in Catalytic Oxidations, vol. 97 of Structure and Bonding (Springer, Berlin 2000), pp. 145-177.

27. A carefully controlled $x$-ray exposure was used to collect the diffraction data so that the dose absorbed by the crystal amounted to $\sim 1 \%$ of the Henderson limit, i.e., $\sim 2.1 \times 10^{5}$ gray (Gy).

28. These rearrangements also bring the $\mathrm{N}_{\varepsilon}$ atom of $\mathrm{His}^{119}$ (the only histidine coordinating the iron through $\mathrm{N}_{\delta}$ ) to a close distance $(3.5 \AA)$ to the distal oxygen of the hydroperoxo moiety.

29. D. Tobi, I. Bahar, Proc. Natl. Acad. Sci. U.S.A. 102, 18908 (2005).

30. M. R. Bukowski, H. L. Halfen, T. A. van den Berg, J. A. Halfen, L. Que, Angew. Chem. Int. Ed. 44, 584 (2005).

31. T. C. Brunold, E. I. Solomon, J. Am. Chem. Soc. 121, 8277 (1999).

32. Coordinates and structure factor amplitudes of the

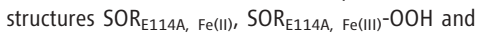
$\mathrm{SOR}_{\text {wt, }} \mathrm{Fe}(\mathrm{II})$ have been deposited in the RCSB Protein Data Bank with pdb codes 2ji2, 2ji3, and 2ji1, respectively. The ESRF is acknowledged for continuous support of our methodological developments. We are grateful to the ESRF beamline staff at ID14-1, ID14-2, ID14-4, and ID29 for their expert assistance; T. Mattioli and C. Mathé for insightful discussions and for providing us with resonance Raman spectra on the E114A SOR mutant; C. Mathevon for help in sample preparation; M. F. Field and L. David for help with the $\mathrm{p} K_{\mathrm{a}}$ calculations; ]. C. Fontecilla for reading the manuscript; and $\mathrm{H}$. Jouve and J.-P. Mahy for providing us with $\mathrm{H}_{2}{ }^{18} \mathrm{O}_{2}$. G.K. acknowledges support by a European Molecular Biology Organization long-term fellowship, and D.B. acknowledges support by the Contrat de Plan Etat-Région and the Action Concertée Incitative programs from the French Ministry of Research. V.N. acknowledges support by the Toxicologie Nucléaire program from the CEA

\section{Supporting Online Material}

www.sciencemag.org/cgi/content/full/316/5823/449/DC1

Materials and Methods

SOM Text

Figs. S1 to S3

Tables S1 to S5

References

Movie S1

15 December 2006; accepted 12 March 2007

10.1126/science. 1138885

\section{Crystal Structures of $\mathrm{Fe}^{2+}$ Dioxygenase Superoxo, Alkylperoxo, and Bound Product Intermediates}

\section{Elena G. Kovaleva and John D. Lipscomb*}

We report the structures of three intermediates in the $\mathrm{O}_{2}$ activation and insertion reactions of an extradiol ring-cleaving dioxygenase. A crystal of $\mathrm{Fe}^{2+}$-containing homoprotocatechuate 2,3dioxygenase was soaked in the slow substrate 4-nitrocatechol in a low $\mathrm{O}_{2}$ atmosphere. The $\mathrm{x}$-ray crystal structure shows that three different intermediates reside in different subunits of a single homotetrameric enzyme molecule. One of these is the key substrate-alkylperoxo- $\mathrm{Fe}^{2+}$ intermediate, which has been predicted, but not structurally characterized, in an oxygenase. The intermediates define the major chemical steps of the dioxygenase mechanism and point to a general mechanistic strategy for the diverse 2-His-1-carboxylate enzyme family.

A

erobic life is possible because $\mathrm{O}_{2}$ must be activated before it will react rapidly with most biological molecules, which prevents indiscriminate oxidation. Oxygenase enzymes have evolved numerous chemical strategies to selectively effect this activation so that 